\title{
AN ARITHMETICAL INVERSION PRINCIPLE
}

\section{BY ECKFORD COHEN}

Communicated by G. B. Huff, July 6, 1959

Let $f(n, r)$ represent an even function of $n(\bmod r)$; that is, $f(n, r)$ $=f((n, r), r)$ for all integers $n$ and a positive integral variable $r$. The following inversion relation is proved in [2]. If $r=r_{1} r_{2}$ and $f(n, r)$ is even $(\bmod r)$, then

$$
g\left(r_{1}, r_{2}\right)=\sum_{d \mid r_{1}} f\left(\frac{r_{1}}{d}, r\right) \mu(d) \rightleftarrows f(n, r)=\sum_{d \mid r} g\left(d, \frac{r}{d}\right),
$$

where $\mu(r)$ denotes the Möbius function. This relation can be easily verified on the basis of the definition of even function $(\bmod r)$ and the characteristic property of $\mu(r)$,

$$
\sum_{d \mid r} \mu(d)=\epsilon(r) \equiv \begin{cases}1 & (r=1), \\ 0 & (r>1) .\end{cases}
$$

We now state a generalization of (1). Let $\xi(r)$ and $\eta(r)$ be arithmetical functions satisfying

$$
\sum_{d \delta=r} \xi(d) \eta(\delta)=\epsilon(r) .
$$

The following theorem can be proved in the same manner as (1), with (3) used in place of (2).

THEOREM 1. If $r=r_{1} r_{2}$ and $f(n, r)$ is even $(\bmod r)$, then

$$
g\left(r_{1}, r_{2}\right)=\sum_{d \mid r_{1}} f\left(\frac{r_{1}}{d}, r\right) \eta(d) \rightleftarrows f(n, r)=\sum_{d \delta=(n, r)} g\left(d, \frac{r}{d}\right) \xi(\delta) .
$$

Clearly (4) reduces to (1) in case $\xi(r)=1, \eta(r)=\mu(r)$. The case $\xi(r)=\mu(r), \eta(r)=1$ yields the following dual of (1).

THEOREM 2. If $r=r_{1} r_{2}$ and $f(n, r)$ is even $(\bmod r)$, then

$$
g\left(r_{1}, r_{2}\right)=\sum_{d \mid r_{1}} f\left(\frac{r_{1}}{d}, r\right) \rightleftarrows f(n, r)=\sum_{d \delta=(n, r)} g\left(d, \frac{r}{d}\right) \mu(\delta) .
$$

An immediate consequence of Theorem 2 is

Corollary 2.1. For every arithmetical function $g\left(r_{1}, r_{2}\right)$ of two positive integral variables $r_{1}, r_{2}$, there exists a uniquely determined even function (mod $r), f(n, r)$, such that $g\left(r_{1}, r_{2}\right)$ is expressible as a divisor sum (5) with respect to $f(n, r)$. 
The relation (1) is applied in [2] to give a new proof of the Anderson-Apostol generalization [1] of the Hölder formula,

$$
\frac{\phi(r) \mu(m)}{\phi(m)}=\sum_{d \mid(n, r)} d \mu\left(\frac{r}{d}\right), \quad \quad\left(m=\frac{r}{(n, r)}\right),
$$

where $\phi(r)$ represents the Euler $\phi$-function. The following analogue of the generalized Hölder relation can be proved in a similar manner, with (5) replacing (1) in the proof.

Let $g(r)$ and $h(r)$ denote arithmetical functions, and define

$$
f(n, r)=\sum_{d \delta=(n, r)} h(d) g\left(\frac{r}{d}\right) \dot{\mu}^{2}\left(\frac{r}{d}\right) \mu(\delta), \quad F(r)=f(0, r) .
$$

TheOREm 3. If $g(r)$ is multiplicative and $h(r)$ is completely multiplicative, and if for all primes $p, h(p) \neq 0, g(p) \neq h(p)$, then

$$
\frac{F(r) g(m) \mu^{2}(m)}{F(m)}=f(n, r), \quad\left(m=\frac{r}{(n, r)}\right) .
$$

Application of (8), with $h(r)=r, g(r)=1$, in connection with the Dedekind-Liouville formula, $\phi(r)=\sum_{d \mid r} d \mu(r / d)$, yields the following analogue of Hölder's formula (6):

Corollary 3.1.

$$
\frac{\phi(r) \mu^{2}(m)}{\phi(m)}=\sum_{d e=r ; d \delta=(n, r)} d \mu^{2}(e) \mu(\delta), \quad\left(m=\frac{r}{(n, r)}\right) .
$$

Similarly, with $h(r)=1, g(r)=\mu(r) / \phi(r)$ in (8) it follows, on applying Landau's identity, $r / \phi(r)=\sum_{d \mid r} \mu^{2}(d) / \phi(d)$, that

Corollary 3.2.

$$
\frac{(n, r) \mu(m)}{\phi(r)}=\sum_{d e=r ; d \delta=(n, r)} \frac{\mu(e) \mu(\delta)}{\phi(e)}, \quad\left(m=\frac{r}{(n, r)}\right) .
$$

Other potentially useful relations can be derived in a similar manner.

\section{BiBLIOGRAPHY}

1. D. R. Anderson and T. M. Apostol, The evaluation of Ramanujan's sum and generalizations, Duke Math. J. vol. 20 (1953) pp. 211-216. Math.

2. Eckford Cohen, Arithmetical inversion formulas, to appear in the Canad. J.

UNIVERSITY OF TENNESSEE 